\title{
Treatment Strategies in Gastric Cancer
}

Hans-Joachim Meyer and Hansjochen Wilke

\section{SUMMARY}

Background: Gastric cancer has become less common but remains among the leading causes of death from cancer, with a 5-year survival rate of only $20 \%$ to $25 \%$. Although diagnostic techniques have improved, most patients with gastric cancer in the Western world (unlike in some Asian countries) already have locally advanced disease when diagnosed and may thus need not only surgery, but also perioperative chemotherapy and/or radiotherapy.

Method: Articles published from 2000 to 2010 and containing the terms "gastric cancer," "surgery," and "chemotherapy" in combination with "review" or "randomized trial" were retrieved by a search in the Cochrane Library and Medline databases and selectively reviewed.

Results: Complete (R0) resection of the tumor remains the standard treatment whenever possible. Complete endoscopic resection suffices only in special types of carcinoma that are confined to the gastric mucosa. Depending on the histological findings, either a subtotal distal gastrectomy or a total (perhaps extended total) gastrectomy can be performed. The long-term benefit of systematic D2 lymphadenectomy has now been shown in a randomized trial: the rates of tumor-related death and of local or regional recurrence were found to be significantly lower with D2 than with D1 lymphadenectomy. Multimodal treatment strategies including perioperative chemotherapy and/or radiotherapy can further improve local and regional tumor control and lessen the rate of systemic metastasis.

Conclusion: The standardization of surgical procedures lowered the operative risk in the treatment of gastric cancer. Patients with locally advanced disease can now derive additional benefit from perioperative chemotherapy with an increase of the 5 -year survival rates of more than $10 \%$.

- Cite this as:

Meyer HJ, Wilke H: Treatment strategies in gastric cancer. Dtsch Arztebl Int 2011; 108(41): 698-706.

DOI: 10.3238/arztebl.2011.0698

Klinik für Allgemein- und Viszeralchirurgie Städtisches Klinikum Solingen: Prof. Dr. med. Meyer

Klinik für Internistische Onkologie, Hämatologie mit Zentrum für Palliativmedizin Kliniken Essen-Mitte: Prof. Dr. med. Wilke
D astric carcinoma remains a therapeutic challenge. Although its overall incidence around the world is declining, more than 130000 new cases of gastric carcinoma were diagnosed in Europe in 2000 (1). The declining incidence is mainly attributable to better food preservation and better nutrition, albeit with wide geographical variation. The German Federal Statistical Office reports that 10510 persons died of a newly diagnosed malignant disease of the stomach in 2008. Gastric carcinoma remains one of the most common causes of death from cancer.

\section{Learning objectives}

This article should enable the reader to

- gain an overview of the indications and the inclusion and exclusion criteria for the surgical treatment of gastric carcinoma, and

- become acquainted with the multimodal treatment strategies for patients with gastric carcinoma and with the scientific evidence underlying them.

In the Western world, most cases of gastric carcinoma are diagnosed in the locally advanced or disseminated stage. In Europe, only $10 \%$ to $15 \%$ of cases are diagnosed in an early stage. The situation is different in Asia: In Japan, for example, the corresponding figure is over $50 \%$.

Despite the reduction of surgical morbidity and mortality with standardized surgical techniques, the overall prognosis remains poor: In the Western world, the 5-year survival rates in all tumor stages are only $20 \%$ to $25 \%$, with a median survival time of about 24 months. Nonetheless, patients in certain subgroups can live longer if they are given stage-directed multimodal treatment, particularly in hospitals with high case numbers $(2,3)$.

In this article, we present current treatment strategies for adenocarcinoma of the stomach and of the gastroesophageal junction on the basis of a selective review of the literature, including published clinical trials and

\section{Gastric carcinoma}

In the Western world, the 5-year survival rate of gastric carcinoma is $20 \%$ to $25 \%$, with a median survival time of about 24 months. 
Cochrane analyses. Current guideline recommendations are also included.

\section{Pre-treatment diagnostic assessment and tumor staging}

Once the diagnosis is confirmed, the degree of tumor spread is ascertained as precisely as possible to serve as the basis for individual treatment planning. Esophagogastroduodenoscopy (video-endoscopy), with targeted removal of multiple biopsy specimens and direct visualization of the site and extent of the tumor, remains the most important component of primary diagnostic assessment (4, 5). Endosonography, computerized tomography, and (where appropriate) magnetic resonance imaging are all comparably useful techniques for determining the degree of local and regional tumor spread (6). Endosonography should be part of the staging assessment whenever treatment is to be provided with curative intent but is not absolutely required before local endoscopic treatment. If there is difficulty determining whether the carcinoma is mucosal or submucosal, as is often the case, the primary approach should consist of so-called diagnostic endoscopic resection. Endoscopic ultrasonography enables the differentiation of categories T1/2 from T3/4. To detect potential distant metastases, computerized tomography (CT) of the abdomen should be performed, and a chest CT as well if the primary tumor involves the gastroesophageal junction. Exploratory laparoscopy is advisable only for locally advanced disease (categories T3/4), with the main purpose of detecting small subcapsular liver metastases or localized peritoneal carcinosis. In such cases, exploratory laparoscopy improves the precision of staging, yielding a correction of the pre-laparoscopic staging in $30 \%$ of cases or more; this can, of course, have major implications for treatment (5).

\section{Current treatment strategies}

The therapeutic recommendations for gastric carcinoma are based on the results of randomized trials and Cochrane analyses as well as on expert consensus. In some areas, evidence on the highest level is not available for the formulation of recommendations.

The main prognostic factors in gastric carcinoma, both tumor- and patient-related, can already be determined at the time of diagnosis. These include

- the tumor stage,

- the site of the tumor, and

- the patient's accompanying diseases, if any.

\section{FIGURE 1}

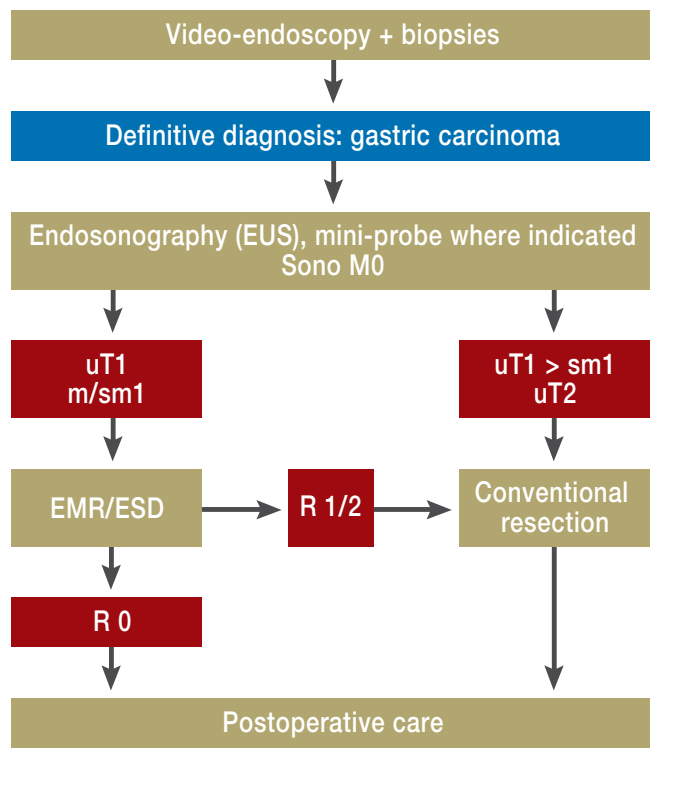

Flowchart for the diagnosis and treatment of early gastric carcinoma

EMR Endoscopic mucosal resection

ESD Endoscopic submucosal dissection

Sono M0 No sonographic evidence of distant metastases

uT1 m/sm1 Endosonographic definition of infiltration into the muscularis mucosae or submucosa

UT2 Endosonographic definition of infiltration into the muscularis propria

RO No residual tumor

R1/2 Micro-/macroscopic residual tumor

The main prognostic factors can only rarely be directly affected by surgical treatment. The main way to improve outcomes is to achieve R0 resection, i.e., complete removal of the tumor and of its regional lymphatic drainage $(2,7)$. Endoscopic procedures can also be employed with curative intent in the treatment of mucosal carcinoma (8). For tumors that have not yet spread beyond the locoregional stage $(\mathrm{T} 1 \mathrm{~b} / 2)$, surgery is the primary treatment (Figure 1). For tumors in more advanced stages (T3 and above) that have not yet given rise to distant metastases or peritoneal carcinomatosis, the option of perioperative chemotherapy should be considered by an interdisciplinary tumor board. For

\section{Primary diagnostic assessment}

Esophagogastroduodenoscopy (video-endoscopy), with targeted removal of multiple biopsy specimens and direct visualization of the site and extent of the tumor, remains the most important component of primary diagnostic assessment.

\section{Current treatment strategies}

- For tumors that are locally or regionally confined ( $\mathrm{T} 1 \mathrm{~b} / 2)$, surgery is the primary treatment.

- For advanced tumors (T3 and above), perioperative chemotherapy should be considered. 


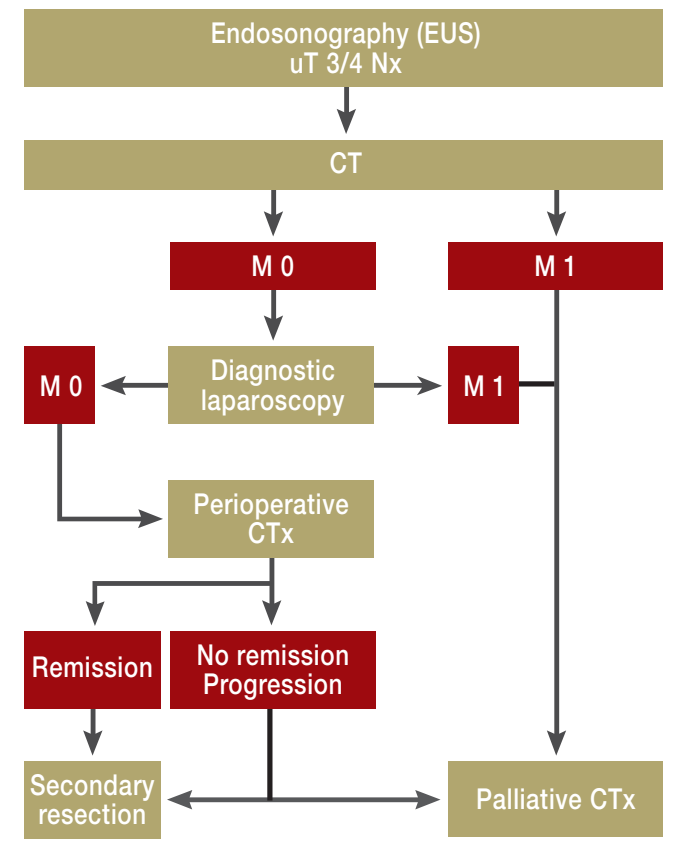

Flowchart for the diagnosis and treatment of advanced gastric carcinoma

uT3/4 Endosonographic definition of infiltration into the subserosa or into adjacent structures

Nx Regional lymph nodes were not assessed

CT Computerized tomography

MO No distant metastases

M1 Distant metastases

CTx Chemotherapy

tumors that have already metastasized, palliative chemotherapy is generally indicated (Figure 2). In the rare cases of major tumor-related complications, such as obstruction, hemorrhage, or perforation, the decision whether to treat with palliative resection or with interventional measures should be taken on an individual basis. When an R1 or R2 resection has been performed, it should be determined whether a second resection with curative intent is feasible; if not, combined chemotherapy and radiation therapy can be given (2-4).

In recent years, an increasing number of reports have highlighted the beneficial effect of high case numbers (per hospital and per surgeon) on treatment outcomes in gastric carcinoma, but there has not been any randomized trial on this question. Although the data are not fully clear, the trend suggests that it is mainly a high case number per hospital that lowers postoperative morbidity and mortality and improves long-term outcomes $(2,9)$.

\section{Surgery}

Complete (R0) resection is the only unimodal treatment with curative intent. The extent of intra- and extraluminal resection is determined according to the basic principles of oncological surgery: a variety of techniques can be used, depending on the particular tumor constellation (see below) and the patient's individual risk factors (7).

\section{Intraluminal resection}

The extent of gastric resection is determined mainly by $(2,7)$

- the site of the tumor,

- the depth of tumor infiltration, and

- the histomorphological classification of the tumor in the Laurén scheme, with consideration of the oral safety margins.

\section{Endoscopic resection}

For local treatment with curative intent in early gastric carcinoma (sometimes in combination with endoscopy and laparoscopy), the key consideration is that of potential lymph node metastases. The rate of metastasis to lymph nodes is $3 \%$ to $6 \%$ in mucosal carcinoma and as high as $30 \%$ in submucosal carcinoma. In a large-scale retrospective study of early gastric carcinoma treated with gastrectomy and meticulous lymph node resection, subgroups were identified in which the observed probability of lymph node metastasis was near zero (10). For more than 15 years, the Japanese approach to patients in one of these subgroups at low risk (i.e., patients with mucosal carcinoma, macroscopic type I or II, diameter less than $20 \mathrm{~mm}$, differentiation G1/2, L0, V0, intestinal carcinoma) has involved endoscopic mucosal resection as a definitive procedure with curative intent after a complete histological survey. There are still no data from randomized trials to support this treatment approach, which has not become established in the Western world because of the low rate of diagnosis of gastric carcinoma in the early stage. It should be carried out only in institutions with the appropriate

\section{Surgery}

Complete $(\mathrm{R} 0)$ resection is the only unimodal treatment with curative intent.

\section{Lymph node metastases}

For local treatment with curative intent in early gastric carcinoma (sometimes in combination with endoscopy and laparoscopy), the key consideration is that of potential lymph node metastases. 


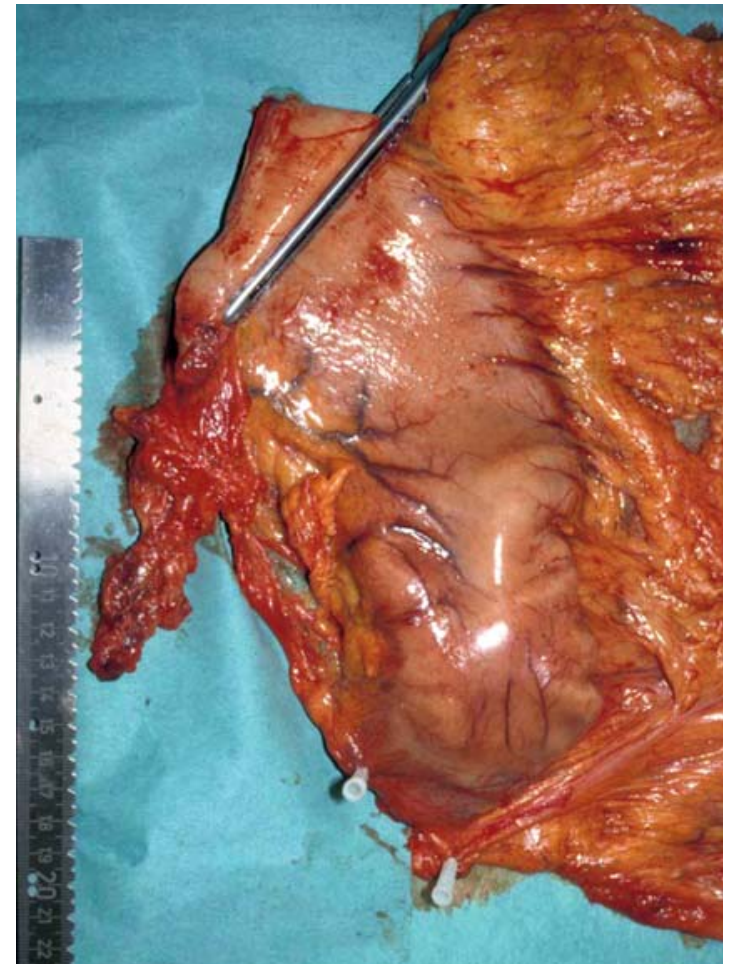

Figure 3: The removed specimen after subtotal distal gastric resection

experience, so that the chance of a cure should not be jeopardized by inadequate treatment. This requirement applies, in particular, to the "extended criteria," up to and including endoscopic submucosal dissection $(3,8)$.

\section{Minimally invasive techniques}

Asian countries have also seen the widest use of laparoscopic resective surgery for gastric carcinoma, including (in recent years) lymphadenectomy via laparoscopy. There have been only a few randomized trials of laparoscopic resection, mostly involving early carcinoma in the distal portion of the stomach. While it can be concluded from recent meta-analyses that minimally invasive partial or total gastrectomy, in accordance with the oncological criteria, is technically feasible, no conclusive judgment about these techniques is possible to date $(3,11)$.

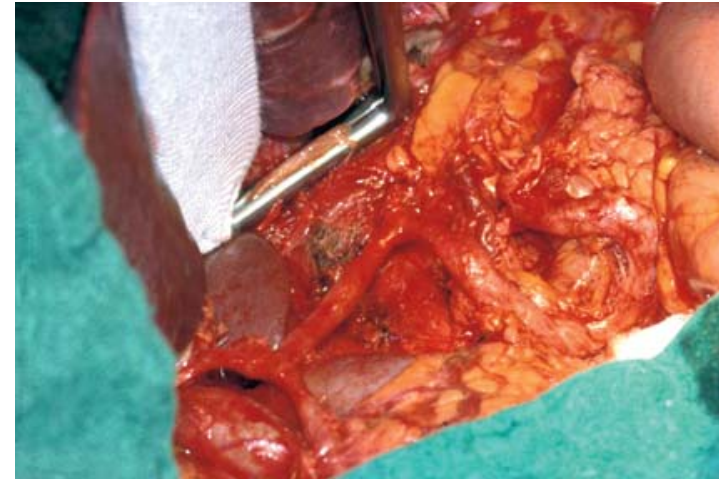

Figure 4: The operative field after systematic D2 lymphadenectomy

\section{Conventional resection}

The definition of $\mathrm{R} 0$ resection, now considered to be standard for stage-appropriate treatment, implies that there is, in principle, no longer any justification for gastrectomy as traditionally performed. Two randomized trials and one observational study have shown that the results of subtotal distal gastrectomy, observing the appropriate oral safety margins when the tumor is located in the lower or middle third of the stomach, are no different from those of total gastrectomy with respect to postoperative morbidity or mortality, or overall survival (12).

In intestinal carcinoma or early diffuse carcinoma, the tumor does not extend beyond its grossly visible limits; in contrast, in advanced diffuse carcinoma, there may be discontinuous growth in areas of the gastric wall that appear grossly normal. The required oral safety margin for $\mathrm{R} 0$ resection is $5 \mathrm{~cm}$ for intestinal carcinoma and $8 \mathrm{~cm}$ in situ for the diffuse type. A practical consequence of this is that subtotal distal gastrectomy is considered oncologically adequate for the treatment of early carcinoma of either type in the distal portion of the stomach, or of advanced intestinal carcinoma in its middle third (Figure 3). In all other cases, total gastrectomy should be performed. In adenocarcinoma of the gastroesophageal junction (Type II/III in the Siewert classification, corresponding to cardial or subcardial carcinoma), total gastrectomy must be extended with transhiatal resection of the distal portion of the esophagus $(2,7)$.

\section{Minimally invasive techniques}

Recent meta-analyses show that minimally invasive partial or total gastrectomy, in accordance with the oncological criteria, is technically feasible.

\section{Resection margins}

The required oral safety margin for $\mathrm{RO}$ resection is $5 \mathrm{~cm}$ for intestinal carcinoma and $8 \mathrm{~cm}$ in situ for the diffuse type. 
TABLE 1

Pre- and postoperative chemotherapy vs. surgery alone in the treatment of gastric carcinoma and adenocarcinoma of the gastroesophageal junction

\begin{tabular}{l|l|l|l|l|l} 
Study & $\begin{array}{l}\text { Treatment } \\
\text { (Patients) }\end{array}$ & T3/T4 (\%) & N2/N3 (\%) & $\begin{array}{l}\text { SR (\%; } \\
5 \text { years) }\end{array}$ & $\begin{array}{l}p \text {-value } \\
(\text { HR })\end{array}$ \\
\hline MAGIC & $\mathrm{S}(\mathrm{n}=253)$ & 63 & 30 & 23 & 0.009 \\
& $\mathrm{~S}+\mathrm{PC}(\mathrm{n}=250)$ & 48 & 16 & 36 & 0.75 \\
\hline FNCLCCl & $\mathrm{S}(\mathrm{n}=111)$ & 68 & $80(\mathrm{~N}+)$ & 24 & 0.002 \\
FFCD & $\mathrm{S}+\mathrm{PC}(\mathrm{n}=113)$ & 58 & $67(\mathrm{~N}+)$ & 38 & 0.69
\end{tabular}

T, deep infiltration of tumor; $\mathrm{N}$, lymph node involvement; $\mathrm{SR}$, survival rate; $\mathrm{HR}$, hazard ratio $\mathrm{S}$, surgery; PC, perioperative chemotherapy

\section{Extraluminal resection}

The extent of extraluminal resection is essentially a function of the removal of locoregional lymphatic drainage areas. In the scheme of the Japanese Research Society for Gastric Cancer, the lymphatic drainage is divided into 16 separate components.

- D1 lymphadenectomy, by definition, consists of removal of the perigastric lymph nodes (nos. 1-6).

- D2 lymphadenectomy additionally includes removal of the suprapancreatic lymph nodes along the great vessels (nos. 7-11).

- In D3 lymphadenectomy, further lymph nodes are removed, e.g., the left para-aortal or retropancreatic nodes.

In gastric carcinoma, unlike other types of cancer, the examination of sentinel lymph nodes with dyes or radionuclides is not yet part of routine clinical practice (2).

The therapeutic value of systematic D2 lymphadenectomy was long debated in the Western world but was considered the gold standard in Japan, in the absence of randomized trials (Figure 4).

Five randomized trials on this subject have now been carried out; the Dutch and British trials, in particular, involved enough cases to yield scientifically relevant data. In these trials, the postoperative morbidity and mortality were significantly higher after D2 lymphadenectomy than after D1 lymphadenectomy, without any improvement of the 5-year overall survival rate. It is important to note, however, that D2 lymphadenectomy was performed in these trials in combination with splenectomy and left pancreatectomy (either as obligate accompaniments, or else at a significantly higher rate than with D1 lymphadenectomy). It was precisely this extension of lymphadenectomy that was found to be an important risk factor for increased postoperative morbidity and mortality $(13,14)$.

Other non-randomized or observational studies of D2 lymphadenectomy without splenectomy or pancreatic resection did not show any increase in mortality; instead, D2 lymphadenectomy was found to improve the clinical outcome in some subgroups, e.g., for tumors in stage II and IIIA in the German Gastric Carcinoma Study (15). These observations led the authors of an earlier Cochrane analysis to conclude that the evidence regarding systematic lymphadenectomy was not conclusive (16).

A trend toward a significant survival advantage from spleen- or pancreas-preserving D2 lymphadenectomy was first discernible in the 11-year survival rates of the Dutch study: These were

- $33 \%$ after D1 lymphadenectomy and

- $47 \%$ after D2 lymphadenectomy (3).

Although no difference in overall survival remained at 15 years, the tumor-associated death rate at 15 years was significantly higher for D1 than for D2 lymphadenectomy (48\% versus 37\%). Furthermore, the locoregional recurrence rate was lower after systematic lymph node resection. These findings imply that spleen- and pancreas-preserving D2 lymphadenectomy is safe and should be recommended as a standard procedure for surgery with curative intent, particularly in institutions with high case numbers, where such operations have already been performed for many years ( 7 , 17).

The available data from clinical trials imply that a further extension of D2 lymphadenectomy to include the left para-aortal nodes, although it is technically feasible without increased morbidity, still does not improve the overall outcome (18).

Splenectomy and/or left pancreatic resection seems necessary only in cases of direct infiltration of these organs by tumor or of potential lymph node metastases to the splenic hilar region. In cases of tumor growth beyond the gastric wall with suspected infiltration of the adjacent organs, multi-organ resection is indicated only if it can be performed as an R0 multi-organ resection, so that the prognosis will be improved (2).

\section{Reconstructive techniques}

In view of the many different surgical techniques available and the low level of the available scientific

\section{An apparent trend}

A trend toward a significant survival advantage from spleen- or pancreas-preserving D2 lymphadenectomy is discernible in the 11-year survival rates of the Dutch study.

\section{Reconstructive techniques}

In view of the many reconstructive techniques available and the low level of the scientific evidence, no particular one can be called the best. 
evidence, no particular reconstructive technique can now be called the best. The current method of first choice after subtotal distal gastric resection is the excluded jejunal loop with creation of a gastrojejunostomy (Roux-en-Y); this method is also the one most commonly used worldwide after total gastrectomy as well. Another option that has been under discussion for decades is that of jejunal interposition including the duodenum or the formation of a pouch; this may be advantageous for the postoperative course. In the end, reconstructive techniques are generally chosen on the basis of the surgeon's personal experience and the patient's prognosis $(2,7)$.

\section{Multimodal treatment}

Perioperative multimodal treatment for gastric carcinoma has been studied in randomized trials; the more recent ones have often included adenocarcinoma of the gastroesophageal junction as well.

\section{Postoperative (adjuvant) chemotherapy}

Multiple meta-analyses comparing the results of surgery with and without adjuvant chemotherapy have found that the former confers a marginal but statistically significant benefit: Survival rates are $4 \%$ to $6 \%$ higher with adjuvant chemotherapy, and patients with advanced tumors stand to benefit most. The single meta-analysis that was based on individual patient data (17 studies, 3838 patients, 5-year survival rate 55.3\% vs. $49.6 \%$ ) did not reveal any significant difference in the survival rates of patients from Asia and the Western world (19).

Thus, because it confers no more than a modest survival advantage that has been documented in the Western world only by meta-analyses, adjuvant chemotherapy for these patients is currently considered a therapeutic option that should be considered in individual cases, e.g., for patients with advanced tumors.

\section{Postoperative (adjuvant) chemotherapy and radiotherapy}

In an American trial involving 556 patients, surgery with postoperative chemotherapy and radiotherapy was compared with surgery alone (20). Adjuvant treatment yielded significant benefit at three years with respect to both disease-free survival (48\% vs. $31 \%$ ) and overall survival ( $50 \%$ vs. $41 \%)$. These results have been called into question, however, because of the inadequate surgical treatment. Thus, there can as yet be no general

\section{TABLE 2}

\section{Reported outcomes after chemotherapy for gastric carcinoma}

\begin{tabular}{l|l|l|l|l|l|l} 
Author & Regimen & $\begin{array}{l}\text { Patients } \\
(\mathrm{n})\end{array}$ & M1 (\%) & RR (\%) & $\begin{array}{l}\text { mPFST } \\
\text { (months) }\end{array}$ & $\begin{array}{l}\text { mST } \\
\text { (months) }\end{array}$ \\
\hline $\begin{array}{l}\text { Kang } \\
(2009)\end{array}$ & XP & 166 & 99 & 41 & 5.6 & 10.5 \\
\hline $\begin{array}{l}\text { Al-Batran } \\
(2008)\end{array}$ & FLO & 156 & 99 & 29 & 5.0 & 9.3 \\
\hline Cunningham & ELF & 108 & 97 & 35 & 5.8 & 10.7 \\
(2008) & ECX & 263 & 79 & 40.7 & 6.2 & 9.9 \\
& EOF & 250 & 77 & 46.4 & 6.7 & 9.9 \\
& EOX & 244 & 77 & 42.4 & 6.5 & 9.3 \\
\hline Van Cutsem & DCF & 221 & 97 & 37 & 5.6 & 9.2 \\
(2006) & CF & 224 & 97 & 25 & 3.7 & 8.6 \\
\hline Dank & IF & 172 & 96 & 31.8 & 5.0 & 9.0 \\
(2008) & CF & 165 & 95 & 26.4 & 4.2 & 8.7 \\
& & & & & & \\
\hline
\end{tabular}

M1, metastasized; RR, remission rate; mPFST, median progression-free survival time; $\mathrm{mST}$, median survival time; XP, capecitabin/cisplatin; CF, cisplatin/5-fluorouracil (5-FU); FLO, 5-FU/folic acid/oxaliplatin; PLF, cisplatin/folic acid/5-FU; ECF, epirubicin/cisplatin/5-FU; ECX, epirubicin/cisplatin/capecitabin; EOF, epirubicin/oxaliplatin/5-FU; EOX, epirubicin/oxaliplatin/5-FU; DCF, docetaxel/cisplatin/5-FU; IF, irinotecan/5-FU

recommendation for postoperative chemotherapy and radiotherapy, although such treatment is an option after surgery without D2 lymphadenectomy.

\section{Pre- and postoperative (perioperative) chemotherapy}

Current interest centers on treatment strategies that can lead to higher rates of $\mathrm{R} 0$ resection, which is considered a determinative prognostic factor. In two randomized trials (the MAGIC trial, with 532 patients, and the FNCLCC/FFCD trial, with 224), surgery with pre- and postoperative chemotherapy was found to improve survival rates markedly in comparison to surgery alone $(21,22)$. These trials included patients with adenocarcinoma of the stomach and gastroesophageal junction (Siewert types I-III) - an acceptable procedure, because the efficacy of chemotherapy is the same for tumors at these two sites.

The perioperative chemotherapy was based on cisplatin and 5-fluorouracil (5-FU) in both trials, without any elevation of postoperative morbidity or mortality. Only about half of all patients for whom postoperative chemotherapy was planned actually received it. In both trials, the 5-year survival rate of surgery combined with

\section{Postoperative adjuvant chemotherapy}

Adjuvant chemotherapy confers a marginal but statistically significant benefit: an improvement in survival rates by $4 \%$ to $6 \%$, with the greatest benefit in patients with advanced tumors.
Postoperative chemotherapy and radiotherapy Postoperative chemotherapy and radiotherapy are generally not recommended, although such treatment is an option after surgery without D2 lymphadenectomy. 
perioperative chemotherapy was higher than that of surgery alone, to a statistically significant extent (36\% vs. $23 \%$ in the MAGIC trial and $38 \%$ vs. $24 \%$ in the FNCLCC/FFCD trial).

These findings, which are summarized in Table 1, lead to the following conclusions:

- Perioperative chemotherapy based on cisplatin and 5-FU increases the survival rates of patients in clinical stages II and III.

- For locally advanced tumors in categories T3 and $\mathrm{T} 4$, perioperative chemotherapy is recommended as the treatment of choice (evidence level 1).

- It is not yet known to what extent the observed benefit was due to the postoperative component of perioperative chemotherapy, if at all. Nonetheless, postoperative chemotherapy is recommended whenever perioperative chemotherapy is to be given (outside of clinical trials as well).

\section{Preoperative chemotherapy and radiation therapy}

In the treatment of "classic" resectable gastric carcinoma, preoperative chemotherapy combined with radiation therapy has not been adequately tested and therefore cannot be recommended, except perhaps in the case of locally advanced adenocarcinoma of the gastroesophageal junction, for which an interdisciplinary consensus favors such treatment (23).

\section{Palliative chemotherapy}

The benefit of systemic drug therapy (chemotherapy) in comparison to best supportive care has been clearly demonstrated, as has the greater efficacy of cytostatic agents in combination rather than as monotherapy. From the findings of the randomized trials that have been carried out in this area, the following conclusions can be derived (Table 2) $(24,25)$ :

- Combination therapy based on platinum and fluoropyrimidine is the treatment of choice for most patients. In clinical trials, this has yielded objective remission rates of $35 \%$ to $40 \%$ and median survival times of 9 to 11 months.

- When it is to be given in combination with platinum derivatives, intravenous 5-FU can be replaced by capecitabin without loss of efficacy.

- Patients whose tumors overexpress the growth factor receptor HER2 (about one-quarter of all patients) live longer if they receive the monoclonal antibody trastuzumab as an additional component of their chemotherapy: In a randomized trial with 594 patients, the median survival was 13.8 months with trastuzumab and 11.1 months without it (25).

\section{Conflict of interest statement}

Prof. Wilke serves on advisory boards for Pfizer, Amgen, and Merck. He receives lecture honoraria from Roche, Sanofi-Aventis, Merck, and Amgen. Prof. Meyer states that he has no conflict of interest.

Manuscript submitted on 19 0ctober 2010, accepted after revision on 6 January 2011.

\section{REFERENCES}

1. Keoghley MRB: Gastrointestinal cancers in Europe. Aliment Pharmacol Ther 2003; 18: 7-30.

2. Grundmann RT, Hölscher AH, Bembenek A, Bollschweiler E, Drognitz 0, Feuerbach S, et al.: Diagnostik und Therapie des Magenkarzinoms - Workflow. Zentralbl Chir 2009; 134: 362-74.

3. Hartgrink HH, Jansen EPM, von Grieken NCT, van de Velde CJH: Gastric Cancer. Lancet 2009; 374: 477-90.

4. Moehler M, Al-Batran SE, Andus T, Anthuber M, Arends J, Arnold D, et al.: S3-Leitlinie „Magenkarzinom” - Diagnostik und Therapie der Adenokarzinome des Magens und oesophagogastralen Übergangs. Z Gastroenterol 2011; 49: 461-531.

5. Englisch-Fritz C, Hünerbein M, Porschen R: Diagnostik beim Magenkarzinom. Onkologe 2008; 14: 332-8.

6. Kwee RM, Kwee TC: Imaging in local staging of gastric cancer: A systematic review. J Clin Oncol 2007; 25: 2107-16.

7. Schuhmacher C, Sendler A, Meyer H-J: Chirurgische Therapie des Magenkarzinoms. Onkologe 2008; 14: 339-49.

8. Rabenstein T, Ell C, Feussner H: Endoskopische Resektion und minimal-invasive Verfahren beim Magenkarzinom. Onkologe 2008; 14 : 350-61.

9. Meyer H-J: The influence of case load and the extent of resection on the quality of treatment outcome in gastric cancer. EJSO 2005: 31: 595-604.

10. Gotoda T, Yanagisawa A, Sasako M, Ono H, Nakanishi Y, Shimoda T, et al.: Incidence of lymph node metastasis from early gastric cancer: estimation with a large number of cases at two large centers. Gastric Cancer 2000; 3: 219-25.

11. Gemmill EH, McCulloch P: Systematic review of minimally invasive resection for gastro-oesophageal cancer. Br J Surg 2007; 94: 1461-7.

12. Bozzetti F, Marubini E, Bonfanti G, Miceli R, Piano C, Crose N, et al.: Total versus subtotal gastrectomy: surgical morbidity and mortality rates in a multicenter Italian randomized trial. The Italian Gastointestinal Tumor Study Group. Ann Surg 1997; 226: 613-20.

13. Bonenkamp JJ, Hermans J, Sasako M, van de Velde CJ, Welvaart K, Songun I, et al.: Extended lymph-node dissection for gastric cancer. N Engl J Med 1999; 340: 908-14.

14. Cuschieri A, Weeden S, Fielding J, Bancewicz J, Craven J, Joypaul $V$, et al.: Patient survival after D1 and D2 resections for gastric cancer: long-term results of the MRC randomized surgical trial Surgical Co-operative Group. Br J Cancer 1999; 79: 1522-30.

\section{Preoperative chemotherapy and radiotherapy}

In the treatment of "classic" resectable gastric carcinoma, preoperative chemotherapy combined with radiation therapy has not been adequately tested and thus cannot be recommended.

\section{Palliative treatment}

The benefit of systemic drug therapy (chemotherapy) in comparison to best supportive care has been clearly demonstrated, as has the greater efficacy of cytostatic agents in combination rather than as monotherapy. 
15. Siewert JR, Böttcher K, Stein HJ, Roder JD and the German Gastric Carcinoma Study Group: Relevant prognostic factors in gastric cancer. Ten-Year results of the German Gastric Cancer Study: Ann Surg 1998; 228: 449-61.

16. McCulloch P, Niita ME, Kazi H, Gama-Rodrigues JJ: Gastrectomy with extended lymphadenectomy for primary treatment of gastric cancer. Br J Surg 2005; 92: 5-13.

17. Songun I, Putter H, Meershoek-Klein Kranenbarg E, Sasako M, van de Velde CJH: Surgical treatment of gastric cancer: 15-year followup results of the randomised nationwide Dutch D1D2 trial. Lancet Oncol 2010; 11: 439-49.

18. Sasako M, Sano T, Yamamoto S, Kurokawa Y, Nashimoto A, Kurita A, et al.: D2 lymphadenectomy alone or with para-aortic nodal dissection for gastric cancer. N Engl J Med 2008; 359: 453-62.

19. The GASTRIC (Global Advanced/Adjuvant Stomach Tumor Research International Collaboration) Group: Benefit of adjuvant chemotherapy for resectable gastric cancer. JAMA 2010; 303: 1729-37.

20. Macdonald JS, Smalley SR, Benedetti J, Hundahl SA, Estes NC, Stemmermann GN, et al.: Chemoradiotherapy after surgery compared with surgery alone for adenocarcinoma of the stomach or gastroesophageal junction. N Engl J Med 2001; 345: 725-30.

21. Cunningham D, Allum WH, Stenning SP, Thompson JN, van de Velde $\mathrm{CJ}$, Nicolson M, et al.: Perioperative chemotherapy versus surgery alone for resectable gastroesophageal cancer. N Engl J Med 2006 355: 11-20.

22. Ychou M, Boige V, Pignon JP, Conroy T, Bouché O, Lebreton G, et al.: Perioperative chemotherapy compared with surgery alone for resectable gastroesophageal adenocarcinoma: An FNCLCC and FFCD multicenter phase III trial. J Clin Oncol 2011; 29: 1715-21.

23. Stahl M, Walz MK, Stuschke M, Lehmann N, Meyer HJ, Riera-Knorrenschild J, et al.: Phase III comparison of preoperative chemotherapy compared with chemoradio-therapy in patients with locally advanced adenocarcinoma of the esophagogastric junction. J Clin Oncol 2009; 27: 851-6.

24. Catalano V, Labianca R, Beretta GD, Gatta G, de Braud F, Van Cutsem E: Gastric cancer. Crit Rev Oncol Hematol 2009; 71: 127-64.

25. Van Cutsem E, Kang YK, Chung HC, Shen L, Sawaki A, Lordick F, et al.: Efficacy results from the ToGA trial: a phase III study of trastuzumab added to standard chemotherapy in first-line human epidermal growth factor receptor 2 (HER2)-positive advanced gastric cancer. $J$ Clin Oncol 2009; 27: 798s (abstract LBA4509).

\section{Corresponding author}

Prof. Dr. med. Hans-Joachim Meyer

Klinik für Allgemein- und Viszeralchirurgie

Städtisches Klinikum Solingen

Gotenstr. 1, 42652 Solingen, Germany

meyer@klinikumsolingen.de
Further information on GME

This article has been certified by the North Rhine Academy for Postgraduate and Continuing Medical Education.

Deutsches Ärzteblatt provides certified continuing medical education (CME) in accordance with the requirements of the Medical Associations of the German federal states (Länder). CME points of the Medical Associations can be acquired only through the Internet, not by mail or fax, by the use of the German version of the CME questionnaire within 6 weeks of publication of the article. See the following website: cme.aerzteblatt.de

Participants in the CME program can manage their CME points with their 15-digit "uniform CME number" (einheitliche Fortbildungsnummer, EFN). The EFN must be entered in the appropriate field in the cme.aerzteblatt.de website under "meine Daten" ("my data"), or upon registration. The EFN appears on each participant's CME certificate.

The solutions to the following questions will be published in issue 49/2011. The CME unit "Hereditary Cardiac Arrhythmias" (issue 37/2011) can be accessed until 28 October 2011.

For issue 45/2011, we plan to offer the topic "Normal Pressure Hydrocephalus."

Solutions to the CME questionnaire in issue 33/2011:

Batra A: The Treatment of Tobacco Dependence.

Solutions: 1e, 2b, 3d, 4e, 5c, 6b, 7b, 8c, 9a, 10d 


\section{Please answer the following questions to participate in our certified Continuing Medical Education program. Only one answer is possible per question. Please select the answer that is most appropriate.}

Question 1

What percentage of European patients with newly diagnosed gastric carcinoma have a tumor in the early stage?
a) $10-15 \%$
b) $20-25 \%$
c) $30-35 \%$
d) $40-45 \%$
e) $50-55 \%$

\section{Question 2}

What is the overall 5-year survival rate of gastric carcinoma in all stages in the Western world?
a) $10-15 \%$
b) $20-25 \%$
c) $30-35 \%$
d) $40-45 \%$
e) $50-55 \%$

Question 3

What type of study is of greatest value in the primary diagnostic evaluation of gastric carcinoma?

a) Computerized tomography

b) Endosonography

c) Esophagogastroduodenoscopy

d) Magnetic resonance imaging

e) Laparoscopy

\section{Question 4}

You are treating a patient with a confirmed diagnosis of gastric carcinoma. The tumor stage has been determined by endosonography to be uT1 $\mathrm{m} / \mathrm{sm} 1$. According to the treatment algorithm, what should your next step be?

a) Endoscopic mucosa resection or submucosal dissection

b) Perioperative chemotherapy and surgical resection

c) Combined chemotherapy and radiotherapy without surgery

d) Pain relief as part of a palliative treatment approach

e) Total surgical resection of the esophagus

\section{Question 5}

What percentage of patients with mucosal carcinoma have lymph node metastases?
a) $0-2 \%$
b) $3-6 \%$
c) $7-10 \%$
d) $11-14 \%$
e) $15-18 \%$

Question 6

Which of the following indicates that the patient is not in the low-risk category for endoscopic resection?
a) Mucosal carcinoma
b) Diameter less than $20 \mathrm{~mm}$
c) Ulcerated early gastric carcinoma
d) Intestinal carcinoma
e) Category LO/NO

\section{Question 7}

What oral safety margin, measured in situ, should be observed in diffuse carcinoma (according to Laurén) in order to achieve a tumor-free proximal resection plane?
a) $4 \mathrm{~cm}$
b) $5 \mathrm{~cm}$
c) $6 \mathrm{~cm}$
d) $7 \mathrm{~cm}$
e) $8 \mathrm{~cm}$

Question 8

In D2 lymphadenectomy, which lymph nodes are removed if the tumor is located in the middle third of the stomach?
a) The perigastric lymph nodes
b) The left para-aortal lymph nodes
c) The retropancreatic lymph nodes
d) The right para-aortal lymph nodes
e) The perigastric and suprapancreatic lymph nodes

Question 9

What is the survival advantage (at 11 years) of D2 lymphadenectomy with preservation of the spleen and pancreas compared to D1 lymphadenectomy?
a) $32 \%$ versus $20 \%$
b) $32 \%$ versus $30 \%$
c) $47 \%$ versus $33 \%$
d) $63 \%$ versus $59 \%$
e) $81 \%$ versus $74 \%$

\section{Question 10}

What is the effect of postoperative adjuvant chemotherapy on survival, compared to surgery alone?

a) Survival rate $10-15 \%$ higher; patients with advanced tumors benefit most

b) Survival rate 1-2\% higher; patients with advanced tumors benefit least

c) Survival rate 4-6\% higher; patients with advanced tumors benefit most

d) Survival rate $26-30 \%$ higher; patients with advanced tumors benefit most

e) Survival rate $41-47 \%$ higher; patients with advanced tumors benefit least 